\title{
“CONECTARSE” A LA SOCIEDAD DE LA INFORMACIÓN Y EL CONOCIMIENTO
}

\author{
"Conectar-se" à sociedade da informação e do conbecimento
}

\begin{abstract}
Ronald Teliz
Doctorando por la Universidad de Boenos Aires, Profesor Adj. en la Licenciatura en Ciencias de la Comunicación (UDELAR) y en el Instituto de Filosofía de la Fac. de Humanidades y Ciencias de la Educación. Universidad de la República - (UDELAR). Montevideo - Uruguay, e-mail: rteliz@adinet.com.uy
\end{abstract}

\section{Resumen}

El desarrollo de las nuevas TIC (Tecnologías de la Información y la Comunicación) determinan las líneas de resolución a la hora de pensar e implementar políticas públicas de comunicación, sobre todo en la época del predominio de la técnica que constituye la llamada "Sociedad de la información y el conocimiento". Por lo tanto, la perspectiva que quiero presentar en este trabajo encuentra que el modelo de comunicación predominante, a la hora de pensar tales políticas, es la visión de la comunicación "transmisiva" y que como consecuencia trae aparejada que las regulaciones sobre el proceso de comunicación entre medios y audiencias, están sostenidas y analizadas sobre una base metafórica predominantemente económica, estratégica y excluyente; en virtud de ello se proponen políticas de comunicación que incluso intentando oponerse a este modelo, sin embargo conservan su lógica. Ello exige una revisión crítica y propuestas alternativas que, de manera preliminar, expongo en el artículo.

Palabras-clave: Teorías de la comunicación; Políticas públicas en comunicación; Sociedad de la información.

\section{Resumo}

O desenvolvimento das novas TIC determinam as linhas de resolução no momento de pensar e implementar políticas públicas de comunicação, sobretudo na época do predominio da técnica que constitui a chamada "Sociedade da informação e do conhecimento". Portanto, a perspectiva apresentada neste trabalho é a de que o modelo de comunicação predominante no momento de pensar tais politicas é a visão da comunicação "transmissiva" o que, consequentemente, traz ajustada que as regulações sobre o processo de comunicação entre meios e audiências, estão sustentadas e analisadas sobre uma base metafórica predominantemente econômica, estratégica e exclusiva; em virtude disso se propõem politicas de comunicação que, inclusive, tentando se opor a este modelo, no entanto conservam sua lógica. Isso exige uma revisão crítica e propostas alternativas que, de maneira preliminar, exponho no artigo.

Palavras-chave: Teorias da comunicação; Politcas públicas em comunicação; Sociedade da informação. 


\section{INTRODUCCIÓN}

Las metáforas no solo han de verse como una figura retórica de transposición del nombre de una cosa a otra, más generalmente permiten ilustrar o transferir a un ámbito de actividad o conocimiento un sentido o descripción que originalmente le pertenece a otro ámbito de actividad. Esta transposición, muchas veces, selecciona, enfatiza, suprime u organiza algunas características del área a la que se desplaza, provocando una modificación en nuestro punto de vista o modo de ver el conjunto de relaciones de dicha área.

El pensamiento metafórico no es, en sí mismo, ni malo ni bueno, simplemente es un lugar común e ineludible que sostiene la comprensión rutinaria o cotidiana de situaciones abstractas y altamente complejas. Como sostiene George Lakoff, hay un verdadero sistema de metáforas que usamos automática e irreflexivamente para entender estas situaciones abstractas y complejas de nuestra vida cotidiana. ${ }^{1}$

En una sociedad fuertemente mediada e influenciada por el desarrollo de las nuevas tecnología de la información y la comunicación (TIC), la metáfora de la "conectividad" en una "Sociedad Red" es un buen ejemplo de esta función metafórica. La conectividad o estar "conectado" se ofrece como un apólogo fundante de una nueva perspectiva para entender y describir la sociedad humana. Recientemente, J. Rifkin ha planteado una metáfora de este estilo para examinar y reconceptualizar los principales cambios estructurales que conforman las bases conceptuales y las organización de esta nueva forma de la sociedad, plantea la próxima era, como una era del Acceso en una sociedad Red. ${ }^{2}$ En las sociedades hipercapitalistas, nos dice, "[1]a vida resulta cada vez más mercantilizada y desaparecen las diferencias entre comunicación, comunión y comercio" (RIFKIN, 2000 , p. 20), agregando más adelante.

La brecha entre los poseedores y los desposeídos es ancha, pero la que existe entre los conectados y los desconectados es aún mayor, [...] Las nuevas redes globales de comunicación digital, debido a que son omnipresentes, tienen el efecto de crear un espacio social nuevo y totalizador (RIFKIN, 2000, p. 25).

Lo que nos está sugiriendo Rifkin es que la comunicación se representa cada vez mas a través de emisores y receptores que superan las distancias físicas mediante vínculos electrónicos; la accesibilidad electrónica y la conformación de redes virtuales sustituyen la presencia física y la transforman en datos, flujo y cantidad de información; la omnipresencia de las redes de comunicación borra la distinción entre comunicar, comunidad y comercio (Aunque parezca solo un juego de palabras).

Desde esta perspectiva, es fácil comprender que con el desarrollo de los medios masivos de comunicación, sobre todo con el ingreso al mercado en los años 80 de las computadoras personales con la integración de texto, imagen y sonido en soporte digital, se haya comenzado a redescribir la comunicación a partir de imágenes como "mundo on-line", "redes virtuales", "cibercultura", "ciberinfraestructura" etc, conformando un espacio de producción e investigación que ha promovido la visión de una profunda reestructura en el espacio público. ${ }^{3}$

En este marco, el objetivo de este artículo es analizar las implicaciones de dos aproximaciones de base metafórica al estudio de los procesos de

\footnotetext{
1 " $\{\mathrm{M}\}$ etaphor is pervasive in everyday life, not just in language but in thought and action. Our ordinary conceptual system, in terms of which we both think and act, is fundamentally metaphorical in nature." LAKOFF, G.; MARK, J. Metaphors we live by. Chigago: University of Chigago Press, 1980.

2 RIFKIN, J. Theage of acces J. New York: Tacher/Putnam Inc, 2000. Versión en español: La época del acceso. Barcelona: Paidós, 2000. La terminología de sociedad de la información y sociedad red y su discusión (entre otros) puede encontrarse en CASTELLS, M. The information age: economy, society, and culture. Blackwell: Cambridge, 1996. v. I. "The Rise of the Network Society”. Versión en español: La Era de la Información. Madrid: Alianza, 1999. (ver nota 6, abajo)

3 Aquí considero "espacio público" de acuerdo con D. Wolton, como "un espacio simbólico donde se oponen y se responden los discursos, la mayor parte contradictorios, mantenidos por los diferentes actores políticos, sociales, religiosos, culturales e intelectuales, que componen una sociedad" WOLTON, D. Penser la communication. Paris: Flammarion, 1997. Glosario; en castellano: Pensar la comunicación. Barcelona: Docencia, 2001) Si bien enfatizo el aspecto simbólico, esto no implica no reconocer los comportamientos concretos de los agentes sociales y los condicionamientos materiales del mismo.
}

Rev. Estud. Comun., Curitiba, v. 9, n. 19, p. 89-96, maio/ago. 2008 
comunicación: el modelo de la transmisión vs. modelo ritual. ${ }^{4}$ La manera de concebir el proceso de comunicación desde una concepción tradicional y predominante lo considera como un mero transporte unidireccional, lineal y mecánico de información (transmisión); por otro lado, se ve el proceso desde la perspectiva de la constitución, representación y legitimación de las creencias compartidas por una comunidad que delinean y confirman una manera particular de ver el mundo (ritual).

Sibien diferentes perspectivas en el estudio de los medios de comunicación han emergido históricamente en base a investigaciones empíricas y teóricas muy disímiles, sin embargo, la concepción acerca de la lógica de los medios de comunicación (masivos) y la respuesta a ellos - por parte de las audiencias - se mueve sobre ciertas "sugerencias" no completamente explícitas. Sugerencias que determinan las líneas de resolución a la hora de pensar e implementar políticas públicas de comunicación, sobre todo en la época del predominio de la técnica que constituye la llamada "Sociedad de la información y el conocimiento." Por lo tanto, la perspectiva que quiero presentar en este trabajo encuentra que el modelo predominante es la visión de la comunicación "transmisiva" y que como consecuencia trae aparejada que las regulaciones sobre el proceso de comunicación entre medios y audiencias, están sostenidas y analizadas sobre una base metafórica predominantemente económica, estratégica y excluyente; en virtud de ello se proponen políticas de comunicación que incluso intentando oponerse a este modelo, sin embargo conservan su lógica.

\section{$\mathbf{I}$}

Veamos algunos ejemplos en los cuales la relación entre comunicación, sociedad-comunidad y comercio (economía) se vuelve enmarañada en el marco de una perspectiva "tecnocomunicativa".

En el 2003, en los Estados Unidos el reporte de The National Science Foundation BlueRibbon Advisory Panel on Cyberinfrastructure, titulado "Revolutionizing Science and Engineering through Cyberinfrastructure" nos dice:

Si la infraestructura es requerida para una economía industrial, entonces podríamos decir que la ciberinfraestructura es requerida para una economía del conocimiento"[énfasis en el original], ...una sociedad digital guiada por la información requiere la recolección, almacenamiento, organización, intercambio y síntesis de enormes volúmenes y amplia variedad de información, y la digitalización de censores análogos de datos y de la información acerca de objetos físicos" por lo tanto requiere de "nuevos paradigmas para la clasificación, representación (e.g. estándares, protocolos, formatos, lenguajes), manipulación y visualización de la información. (NATIONAL SCIENCE FOUNDATION 2003, p. 5). ${ }^{6}$

\footnotetext{
${ }^{4}$ CAREY, J. Communication as culture: essays on media and society. Boston: Unwin Hyman, 1985.

5 En la última década, la expresión "sociedad de la información” se ha consagrado como un término hegemónico que se vincula a las políticas oficiales de los países más desarrollados, además de la coronación que significó honrarlo con una Cumbre Mundial. Los antecedentes del término, sin embargo, datan de décadas anteriores. En 1973, el sociólogo estadounidense Daniel BELL introdujo la noción de la "sociedad de la información" en su libro. The coming of post-industrial society: a venture in social forecasting. New York: Basic Books, 1973, donde formula que el eje principal de ésta será el conocimiento teórico y advierte que los servicios basados en el conocimiento habrán de convertirse en la estructura central de la nueva economía y de una sociedad apuntalada en la información, donde las ideologías resultarán sobrando. Esta expresión reaparece con fuerza en los años 90 , en el contex to del desarrollo de Internet y de las TICs. A partir de 1995, fue incluida en la agenda de las reuniones del G7 (luego G8, donde se juntan los jefes de Estado o gobierno de las naciones más poderosas del planeta). Se ha abordado en foros de la Comunidad Europea y dela OCDE (los treinta países más desarrollados del mundo) y ha sido adoptada por el gobierno de los Estados Unidos, así como por varias agencias de las Naciones Unidas y por el Grupo Banco Mundial. A partir de 1998, fue elegida, primero enla Unión Internacional de Telecomunicaciones y luego en la ONU, como el nombre de la Cumbre Mundial a realizarse en 2003 y 2005. Actualmente se encuentra en debate tal denominación (por motivos en los que no podemos ahondar aquí), se propone como sustituto, por ej: sociedad del conocimiento, sociedad informacional, etc.

${ }^{6}$ National Science Foundation. 2003. Revolutionizing science and engineering through Cyberinfrastructure: report of the National Science Foundation Blue-Ribbon Advisory Panel on Cyberinfrastructure; disponible enhttp:/ /www.communitytechnology.org/ nsf_ci_report/. Traducción mia. Aquí “ciberinfraestructura” refiere a la infraestructura basada en red de computadoras, información y tecnologías de la comunicación. Dicho término emerge como análogo al término infraestructura, en el sentido que este refiere colectivamente a carreteras, puentes, vías y bienes públicos similares, requeridos para el funcionamiento de una economía industrial. Así, el término cyberinfraestructura, en tanto refiere al sistema de información y tecnologías de la comunicación, es requerido para el funcionamiento de la una economía sustentada en el conocimiento.
} 
Desde este punto de vista, la transformación de la economía en una red global tiene un impacto en la organización de la sociedad, se pretende que esta transformación está haciendo emerger (o ya lo ha hecho) una Sociedad de la Información y el Conocimiento. Se argumenta que el cambio fundamental que sostiene tan novedosa forma de la sociedad es un paradigma tecno económico comunicativo, que exige nuevos estándares para manipulary visualizar la información. Esta perspectiva supone que la creación, diseminación y preservación de datos, información y conocimiento en la "época digital", exigen una reconceptualización del soporte tecnocomunicativo de las nuevas tecnologías de la comunicación.

Por otra parte y para ver otro ejemplo más reciente, la Comisión Europea responsable para la Sociedad de la Información y los Medios de la Unión Europea ha presentado ante el Parlamento Europeo la iniciativa "2010 - Una sociedad de la información europea para el crecimiento y el empleo."7

Tras declarar, en el marco de la "estrategia de Lisboa", que es indispensable construir una sociedad de la información plenamente integradora, basada en la generalización de la tecnologías de la información y la comunicación (TIC), agrega:

Las tecnologías de la información y la comunicación contribuyen poderosamente a impulsar el crecimiento y el empleo. A ellas se deben la cuarta parte del crecimiento del PIB de la UE y el $40 \%$ del crecimiento de la productividad. Las diferencias de comportamiento económico entre los países industrializados se explican en gran medida por los niveles de inversión, investigación y utilización de las TIC, así como por la competitividad de las industrias de la sociedad de la información y medios de comunicación. ${ }^{8}$ Los servicios, competencias, medios y contenidos relacionados con las TIC forman una parte creciente de la economía y la sociedad.
Más adelante continúa;

La convergencia digital de los servicios, redes y dispositivos de la sociedad de la información y de los medios de comunicación [énfasis en el original] se está convirtiendo al fin en una realidad cotidiana: las TIC ganarán en inteligencia, seguridad y rapidez, se reducirán de tamaño, podrán estar permanentemente conectadas y serán fáciles de usar, mientras los contenidos se orientan hacia los formatos multimedia tridimensionales.

[...] Apoyándose en un análisis completo de los retos asociados a la sociedad de la información y en una amplia consulta con las partes interesadas sobre iniciativas e instrumentos previos, ${ }^{9}$ la Comisión propone tres prioridades para las políticas europeas de sociedad de la información y medios de comunicación:

i) la construcción de un Espacio único Europeo de la Información que promueva un mercado interior abierto y competitivo para la sociedad de la información y los medios de comunicación;

ii) el refuerzo de la innovación y la inversión en la investigación sobre las TIC con el fin de fomentar el crecimiento y la creación de más empleos y de más de calidad;

iii) el logro de una sociedad europea de la información basada en la inclusión que fomenta el crecimiento y el empleo de una manera coherente con el desarrollo sostenible y que da la prioridad a la mejora de los servicios públicos y de la calidad de vida [énfasis en el original].

\footnotetext{
7 Comisión de las Comunidades Europeas Bruselas, 1.6.2005COM(2005) Comunicación de la Comisión al Consejo, al Parlamento Europeo y al Comité Económico y Social Europeo y al Comité de las Regiones. (Bruselas, 1.6.2005 COM(2005) 229 final “i2010 - Una sociedad de la información europea para el crecimiento y el empleo". Disponible en: < http:// www.csi.map.es/csi/pdf/com_229_i2010_310505_fv_es.pdf $>$. Acceso en: 3 mayo 2008.

8 (sic) Los servicios de la sociedad de la información y las industrias de los medios de comunicación fueron ya descritos en el Libro Verde sobre la convergencia de los sectores de telecomunicaciones, medios de comunicación y tecnologías de la información y sobre sus consecuencias para la reglamentación en la perspectiva de la sociedad de la información de 1998 - COM(97) 623 y, teniendo en cuenta la evolución posterior, en la Comunicación de 2003 sobre el futuro de la política reguladora europea en el sector audiovisual - COM(2003) 784. Estos servicios reflejan la convergencia que se está produciendo entre los servicios de comunicaciones electrónicas, los servicios de la sociedad de la información y los servicios de radiodifusión y la aparición de nuevos servicios de contenidos resultantes de ella.).

9 (sic) A saber, las iniciativas eEurope y la Comunicación sobre el futuro de la política reguladora europea en el sector audiovisual, $\operatorname{COM}(2003)$ 784.p. 4.
} 
A las tres prioridades se le suman cuatro grandes retos planteados por la convergencia digital:

- la velocidad: aumentar la velocidad de los servicios de banda ancha en Europa para entregar contenidos enriquecidos, como el vídeo de alta definición;

- riqueza de los contenidos: incrementar la seguridad jurídica y económica para fomentar los nuevos servicios y los contenidos en línea;

- la interoperabilidad: potenciar los dispositivos y las plataformas capaces de «hablar entre sí», y los servicios que se pueden llevar de una plataforma a otra;

- la seguridad: hacer más segura Internet frente al fraude, los contenidos nocivos y los fallos tecnológicos, para aumentar la confianza de los inversores y consumidores. (COMISIÓN DE LAS COMUNIDADES EUROPEAS BRUSELAS, 2005).

Parece obvio que la Comisión propone un nuevo marco estratégico político para la constitución de una Sociedad de la Información europea sustentada en la promoción de una "economía digital abierta y competitiva", haciendo hincapié en las TIC en tanto que impulsoras de la inclusión y mejora de la calidad de vida.

Pero tal inclusión y mejora de la calidad de vida se remite, según las prioridades definidas, a la conectividad de redes como espacios únicos de información que fomenten el crecimiento económico, el empleo y un desarrollo sustentable. A la vez, se consideran retos: el aumento de la velocidad de conducción de la información, mejora en la definición visual de los contenidos; la seguridad y riqueza de los contenidos (información) que se evalúan desde la perspectiva de la prevención de fallos técnicos de conductibilidad, regulaciones jurídicas que salvaguarden el mercado digital.

Dadas estas características de mercado con las que se considera a las nuevas TIC, no es extraño que se considere un reto potenciar los dispositivos y plataformas capaces de "hablar entre sí" y que de esta interoperabilidad se espere que aumente la confianza entre "inversores" y "consumidores" de información.
Como hemos apuntado más arriba, la estrategia de la Unión Europea es hacer de Europa la primera economía basada en la información y el conocimiento. La tendencia en políticas publicas persigue la conformación de una Sociedad de la Información y el conocimiento, es decir, una sociedad que valora y sitúa el énfasis en la importancia de asegurar que el conocimiento sea compartido y transformado en dominio social. Pero si embargo, en la gran mayoría de los casos, el concepto de "Sociedad de la Información y el conocimiento" va acompañado de un énfasis en la contribución de la información y el conocimiento a la riqueza económica de una nación. Se piensa la información y el conocimiento en términos de "mercado digital abierto y competitivo", por lo que la premisa es que el conocimiento es un bien primario de productividad económica y ha de ser manejado estratégicamente como tal.

Por ello, el énfasis en la comercialización de la información y el conocimiento, no reconoce la complejidad de los mismos e infravalora el conocimiento y el desarrollo tecnológico que no tenga aplicación comercial, dejando de lado lo que éste pueda contribuir a la cohesión, integración social y a políticas públicas en comunicación.

Creo que, finalmente, lo que subyace a esta postura es una concepción del vínculo entre comunicación y sociedad sustentado en una visión tecnocomunicativa, que borra toda distinción entre comunicar, comunidad y comercio; es decir, el predominio de una visión o modelo "transmisivo" de la comunicación.

Visto los ejemplos anteriores, integrémoslos a una visión más general sobre la comunicación.

\section{II}

Si estudiar la comunicación es, entre otras cosas, examinar los procesos sociales efectivos mediante los cuales complicadas formas simbólicas son creadas y median el accionar social en un tiempo histórico determinado (p.e. sistemas simbólicos como la ciencia, el arte, religión, sentido común, ideologías, etc), ¿qué diferencias hay entre estos diversos sistemas simbólicos? ¿cómo son influidos por los cambios provenientes de las nuevas tecnologías de la información y la comunicación? Preguntas que son más fáciles de formular que de responder. Sin 
embargo, como hemos sugerido al comienzo de esta exposición, creo que hay un verdadero sistema de metáforas que usamos automática e irreflexivamente para entender estas situaciones abstractas y complejas de nuestra vida cotidiana. Muchas veces la reflexión teórica se sustenta o genera una posición más general a partir de elaboraciones de estos núcleos metafóricos.

Por lo anterior, creo que la distinción que propone James Carey en Communication as Culture: Essays on Media and Society, ${ }^{10}$ entre el punto de vista (que aquí he venido sugiriendo como metáforas) de la "transmisión" de la comunicación y el punto de vista "ritual," 11 es uno de estos casos de generalización y nos será de utilidad para comprender algunos aspectos de la problemática de la comunicación que venimos tratando, por lo que resumiré brevemente tal distinción.

Para Carey, el punto de vista de la comunicación como transmisión es el más común en nuestra cultura, es el que domina en las culturas industriales y el más frecuente a ser encontrado en las entradas de diccionarios bajo el término comunicación. Se le asocia o es definido por términos tales como "impartir", "enviar", "trasmitir" "o dar información a otros". Se conforma sobre la base de una metáfora espacial o geográfica, se piensa en posiciones a partir de las cuales se transporta de un lugar a otro información. Según Carey, en el siglo XIX el movimiento de personas o bienes y el movimiento de información fue visto como un proceso esencialmente idéntico y ambos agrupados o descriptos por el término común "comunicación". El centro de esta idea de comunicación es el de la transmisión de señales o mensajes a distancia con propósitos de control. Este punto de vista deriva de un viejo sueño humano: el deseo de incrementar la velocidad y los efectos de los mensajes que se trasmiten en el espacio (CAREY, 1985, p. 14-17).

El punto de vista ritual de la comunicación, en cambio, esta dirigido a interacciones que persiguen la finalidad o ideal de preservar la sociedad en el tiempo y no como el acto de conferir o ceder información a través de la extensión de los mensajes en el espacio. Un punto de vista ritual de la comunicación se dirige a consolidar la representación de creencias compartidas por una comunidad.

Si el caso paradigmático de la comunicación bajo el punto de vista de la transmisión es elincremento o expansión de los mensajes a través de la "geografía" (espacio) con fines de control, el caso paradigmático bajo un punto de vista ritual es la ceremonia sagrada que impulsa a las personas a compartir intereses y conformar la membresía de una comunidad. El apelar al término "ritual" si bien sugiere fundamentalmente un aspecto religioso o sagrado de la comunicación, no hay que pensarlo necesariamente en el sentido de sermón, admonición, etc. Lo que sugiere es más bien el aspecto de comunión guiado por la charla o dialogo relajado, el canto melodioso y la ceremonia. Es decir, se resalta el aspecto de construcción y mantenimiento de un mundo cultural significativo y ordenado que puede servir como control y continente de la acción humana, en contraposición a la transmisión de información (CAREY, 1985, p. 18-19).

Como ejemplo de ambas concepciones, Carey sugiere que, si uno examina un periódico bajo el punto de vista de la transmisión se lo verá como un instrumento para propagar o extender noticias y conocimientos embalados en amplios paquetes que permiten llegar a grandes distancias, y las interrogantes que genera se dirigen a los efectos sobre la audiencia, en el sentido de que las noticias pueden iluminar $\mathrm{u}$ obscurecer ciertos hechos, cambiar o volver rígidas ciertas actitudes, generar credibilidad o dudas, ganar información, etc. En cambio, desde un punto de vista ritual de la comunicación se focalizarán diferentes rangos de problemas al examinar un periódico, por ejemplo, se le prestará mayor atención o cuidado a aquellas cosas que afectan a la masa, que delinean y confirman un particular punto de vista sobre el mundo. Lo que portan las noticias no es visto como

\footnotetext{
${ }^{10}$ CAREY, James. Communication as culture: essays on media and society. Boston: Unwin Hyman, 1985.

${ }^{11}$ Si bien aquí tomaré la distinción formulada por Carey, bien podría tomarse una distinción similar proveniente de otros marco teóricos, p.e. la de D. Wolton entre "comunicación normativa" y "comunicación funcional" (Penser la communicatión. Paris: Flammarion, 1997. Versión castellana. Pensarla Comunicación. Docencia: Buenos Aires, 2001) que, creo, tiene parentesco con la distinción aquí planteada. Con un poco de esfuerzo se puede encontrar una distinción similar en J. Habermas y su teoría de la acción comunicativa (distinción entre acción comunicativa y acción estratégica. HABERMAS. J. Teoría de la acción comunicativa. Madrid: Taurus, 1987).
}

Rev. Estud. Comun., Curitiba, v. 9, n. 19, p. 89-96, maio/ago. 2008 
pura información, sino que representan o retratan fuerzas que contienden en el mundo. Además, los lectores, a su modo, se comprometen con diversos roles o focos dramáticos de esa contienda.

Bajo el punto de vista ritual, entonces, las noticias no son consideradas información sino drama, no describen el mundo sino que representan o delinean un campo de confrontación de focos dramáticos y acción, que convocan a nuestra participación sobre la base de asumir roles sociales alineados con los del campo (CAREY, 1985, p. 20-21).

Ahora bien, si aceptamos la distinción propuesta por Carey y la proyectamos sobre el espacio público, espacio donde se constituye "el intercambio discursivo de posiciones razonables sobre los problemas de interés general... y donde los individuos más o menos autónomos son capaces de formarse una opinión," 12 no es lo mismo considerar estrategias o políticas públicas de comunicación que lo consideren un mero espacio común de intercambios y comercio, que considerarlo un espacio simbólico donde se confrontan posiciones simbólicas y se constituyen roles.

Creo que es fácil sostener, que si tomamos la noción de Sociedad de la Información y el Conocimiento sustentada en perspectivas que surgen de informes como el de la National Science Foundation o el de la Comisión de la Unión Europea (mencionados arriba) estamos viendo el vínculo entre comunicación y sociedad sustentado en una visión tecnocomunicativa, que borra toda distinción entre comunicar, comunidad y comercio; es decir, aceptar el predominio de una visión o punto de vista "transmisivo" de la comunicación. Que tiene como primera consecuencia, reducir el espacio público a un mero espacio común de intercambios comerciales, donde las regulaciones y desregulaciones operan estratégicamente guiadas por leyes económicas, justamente leyes más o menos homogéneas que permiten concebir a la sociedad como un mercado global. Así, desde el punto de vista de la comunicación, la globalización es más un efecto de cierta perspectiva "transmisiva" de la comunicación que de una homogenización real de los contenidos.
Políticas públicas de comunicación que no valoren el aspecto "ritual" de la comunicación, olvidan el sentido en el que ha de entenderse que todo miembro de una comunidad tiene un derecho inalienable a la información y a la expresión, ya que su acceso a los mismos exige también acceso a los diversos modos de pensar acerca de la información y el conocimiento.

\section{CONCLUSIÓN}

Las nuevas Tecnologías de la Información y la Comunicación están experimentando un gran cambio, nuevas direcciones e inversiones se originan en corporaciones que provienen de otras áreas de actividad social (deportes, entretenimiento, hotelería, etc) y por supuesto han atraído la mirada e intervención sobre ellos de políticas públicas de comunicación. De esta turbulencia, se supone esta surgiendo o ha surgido un nuevo orden y nuevas formas de regulación a escala mundial.

La alta velocidad de las redes de información en conjunción con la compresión de datos que permite trasmitir imagen y sonido con economía y eficacia, ha expandido el universo de la comunicación. Ello ha motivado el incremento en el foco de búsqueda de modelos más complejos que implican redefinir el concepto de comunicación, desde esta perspectiva los medios masivos y las TICs parecen jugar un rol central en el proceso de análisis, construcción y desarrollo de nuevas aproximaciones y metodologías necesarias para esta nueva "época, a la que normalmente se la concibe como "Sociedad de la Información y el conocimiento". No hay ninguna objeción a esta genuina expansión de los canales de comunicación, tanto a nivel local como global, sin embargo, creo que estos canales deben ser considerados en todos sus aspectos, respondiendo a necesidades educativas, de salud y necesidades culturales de un amplio rango. Pero la realidad es que, como tantas otras cosas en nuestra sociedad contemporánea, la información y el conocimiento se han vuelto un bien económico a la venta,

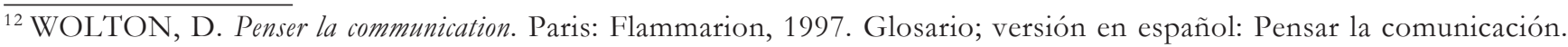
Traducción de José A Alvarez. Buenos Aires: Docencia, 2001. 
disponible en un "mercado digital abierto y competitivo", por lo que se han vuelto un bien primario de productividad económica en desmedro de su función simbólica de conformación e integración social. Con ello, a la vez, se reduce nuestra concepción del valor de la comunicación como factor de integración social a su costado puramente "transmisivo", identificándola con el libre flujo de información que habría que resguardar y potenciar.

\section{REFERENCIAS}

BELL, D. The coming of post-industrial society: a venture in social forecasting. New York: Basic Books, 1973.

CAREY, J. Communication as culture: essays on media and society. Boston: Unwin Hyman, 1985.

CASTELLS, M. The information age: economy, society, and culture. Blackwell: Cambridge, 1996. v. 1. "The Rise of the Network Society"; versión en español: La Era de la Información Versión en español de: Carmen Matinez Gimeno. Madrid: Alianza, 1999.

COMISIÓN DE LAS COMUNIDADES EUROPEAS BRUSELAS. 1.6.2005 COM (2005) Comunicación de la Comisión al Consejo, al Parlamento Europeo y al Comité Económico y Social Europeo y al Comité de las Regiones. (Bruselas, 1.6.2005 $\operatorname{COM(2005)} 229$ final “i2010 - una sociedad de la información europea para el crecimiento y el empleo." Disponible en: <http: / / www.csi.map.es/csi/pdf/ com_229_i2010_310505_fv_es.pdf $>$. Acceso en: 3 mayo 2008
HABERMAS. J. Teoría de la acción comunicativa. Madrid: Taurus, 1987.

LAKOFF, G.; MARK, J. Metaphors we live by. Chicago: University of Chicago Press, 1980.

NATIONAL SCIENCE FOUNDATION. Revolutionizing science and engineering through cyberinfrastructure: report of the national science foundation blue-ribbon advisory panel on cyberinfrastructure. 2003. Disponible en: <http://www.communitytechnology.org/ nsf_ci_report>. Acceso en: <http:// dlist.sir.arizona.edu/897/>. Acceso en: 3 mayo 2008.

RIFKIN, J. The age of acces. New York: J. Tacher/Putnam, 2000; versión en español: La época del Acceso Paidós. Traducción de J. Francisco lvarez y David Teira. Barcelona: Ibérica, 2000.

WOLTON, D. Penser la communication. Paris: Flammarion, 1997. Glosario; versión en español: Pensar la comunicación. Traducción de José A Alvarez. Buenos Aires: Docencia, 2001.

Recebido: 12/05/2008

Received: 05/12/2008

Aprovado: 19/05/2008 Approved: 05/19/2008 